\title{
Epidemiology of hospital-acquired pneumonia: Results of a Central European multicenter, prospective, observational study compared with data from the European region
}

\author{
Tomas Herkel ${ }^{\mathrm{a}}$, Radovan Uvizl ${ }^{\mathrm{a}}$, Lenka Doubravska ${ }^{\mathrm{a}}$, Milan Adamus ${ }^{\mathrm{a}}$, Tomas Gabrhelik ${ }^{\mathrm{b}}$, Miroslava Htoutou Sedlakovac, \\ Milan Kolar', Vojtech Hanulikc, Vendula Pudova', Katerina Langovad, Roman Zazulae, Tomas Rezace, Michal Moravece, \\ Pavel Cermak ${ }^{f}$, Pavel Sevcik ${ }^{g, h}$, Jan Staseki, Jan Malaskai, Alena Sevcikovaj, Marketa Hanslianovaj, Zdenek Turekk, \\ Vladimir Cerny ${ }^{k,}$, Pavla Paterova ${ }^{m}$
}

Background. Hospital-acquired pneumonia (HAP) is associated with high mortality. In Central Europe, there is a dearth of information on the prevalence and treatment of HAP. This project was aimed at collecting multicenter epidemiological data on patients with HAP in the Czech Republic and comparing them with supraregional data.

Methods. This prospective, multicenter, observational study processed data from a database supported by a Czech Ministry of Health grant project. Included were all consecutive patients aged 18 and over who were admitted to participating intensive care units (ICUs) between 1 May 2013 and 31 December 2014 and met the inclusion criterion of having HAP. The primary endpoint was to analyze the relationships between 30-day mortality (during the stay in or after discharge from ICUs) and the microbiological etiological agent and adequacy of initial empirical antibiotic therapy in HAP patients.

Results. The group dataset contained data on 330 enrolled patients. The final validated dataset involved 214 patients, 168 males (78.5\%) and 46 females (21.5\%), from whom 278 valid lower airway samples were obtained. The mean patient age was 59.9 years. The mean APACHE II score at admission was 21 . Community-acquired pneumonia was identified in 13 patients and HAP in 201 patients, of whom 26 (12.1\%) had early-onset and 175 (81.8\%) had late-onset HAP. Twenty-two bacterial species were identified as etiologic agents but only six of them exceeded a frequency of detection of 5\% (Klebsiella pneumoniae 20.4\%, Pseudomonas aeruginosa $20.0 \%$, Escherichia coli 10.8\%, Enterobacter spp. $8.1 \%$, Staphylococcus aureus $6.2 \%$ and Burkholderia cepacia complex 5.8\%). Patients infected with Staphylococcus aureus had significantly higher rates of early-onset HAP than those with other etiologic agents. The overall 30-day mortality rate for HAP was $29.9 \%$, with $19.2 \%$ mortality for early-onset HAP and $31.4 \%$ mortality for late-onset HAP. Patients with late-onset HAP receiving adequate initial empirical antibiotic therapy had statistically significantly lower 30 -day mortality than those receiving inadequate initial antibiotic therapy ( $23.8 \%$ vs $42.9 \%)$. Patients with ventilatorassociated pneumonia (VAP) had significantly higher mortality than those who developed HAP with no association with mechanical ventilation (34.6\% vs $12.7 \%$ ). Patients having VAP treated with adequate initial antibiotic therapy had lower 30 -day mortality than those receiving inadequate therapy ( $27.2 \%$ vs $44.8 \%)$.

Conclusions. The present study was the first to collect multicenter data on the epidemiology of HAP in the Central European Region, with respect to the incidence of etiologic agents causing HAP. It was concerned with relationships between 30-day patient mortality and the type of HAP, etiologic agent and adequacy of initial empirical antibiotic therapy.

Key words: hospital-acquired pneumonia, mortality, HAP, VAP, antibiotic therapy

Received: December 15, 2015; Accepted: March 4, 2016; Available online: March 17, 2016 http://dx.doi.org/10.5507/bp.2016.014

\footnotetext{
${ }^{a}$ Department of Anesthesiology and Intensive Care Medicine, Faculty of Medicine and Dentistry, Palacky University Olomouc and University Hospital Olomouc, Czech Republic

${ }^{b}$ Department of Anesthesiology, T. Bata Hospital, Zlin, Czech Republic

'Department of Microbiology, Faculty of Medicine and Dentistry, Palacky University Olomouc and University Hospital Olomouc, Czech Republic

${ }^{d}$ Department of Medical Biophysics, Faculty of Medicine and Dentistry, Palacky University Olomouc, Czech Republic

${ }^{e}$ Department of Anesthesiology and Intensive Care Medicine, First Faculty of Medicine, Charles University in Prague and Thomayer Hospital Prague, Czech Republic

fDepartment of Microbiology, Thomayer Hospital Prague, Czech Republic

${ }^{g}$ Department of Intensive Care Medicine and Forensic Studies, Faculty of Medicine, University of Ostrava, Czech Republic

${ }^{h}$ Department of Anesthesiology and Intensive Care Medicine, University Hospital Ostrava, Czech Republic

'Department of Anesthesiology and Intensive Care Medicine, Faculty of Medicine, Masaryk University in Brno and University Hospital Brno, Czech Republic

'Department of Microbiology, Faculty of Medicine, Masaryk University in Brno and University Hospital Brno, Czech Republic ${ }^{k}$ Department of Research and Development, Department of Anesthesiology and Intensive Care Medicine, Faculty of Medicine in Hradec Kralove, Charles University in Prague and University Hospital Hradec Kralove, Czech Republic
} 
'Department of Anesthesia, Pain Management and Perioperative Medicine, Dalhousie University, Halifax, Canada

${ }^{m}$ Department of Microbiology, Faculty of Medicine in Hradec Kralove, Charles University in Prague and University Hospital Hradec Kralove, Czech Republic

Corresponding author: Radovan Uvizl, e-mail: radovan.uvizl@seznam.cz

\section{INTRODUCTION}

Early and correct identification of the etiologic agent for nosocomial bacterial infection and determination of its susceptibility or resistance to antibiotics are an essential precondition for rational targeted antibiotic therapy. Additionally, adequate selection of empirical antibiotic therapy makes the treatment more successful, shorter and less expensive ${ }^{1,2}$, eventually leading to reduced patient mortality ${ }^{3}$. One of the most common nosocomial infections encountered in intensive care is hospital-acquired pneumonia (HAP). In intensive care patients, HAP accounts for $10-47 \%$ of nosocomial infections ${ }^{4,5}$, with a reported mortality of $20-60 \%$ (ref. ${ }^{6-9}$ ). Most frequently, HAP is associated with invasive airway management and mechanical ventilation, such as the case of ventilator-assisted pneumonia (VAP) $\left(\right.$ ref. $\left.^{10}\right)$. From an epidemiological point of view, there are two types of pneumonia. Communityacquired pneumonia (CAP) develops outside the hospital setting or within $48 \mathrm{~h}$ from admission to hospital of a patient. By contrast, HAP is associated with a stay in a health care facility and is defined as developing within 48 $\mathrm{h}$ or more from admission, but no later than 14 days after discharge of a patient. Clinical and laboratory manifestations of early-onset HAP occur 48 to 96 hours from admission; in late-onset HAP, the time period starts on day 5 after hospital admission ${ }^{11}$. The pathogenesis of HAP is dependent upon the number and virulence of microorganisms entering the lower airways and the patient's reactions - immune, mechanical, humoral or cellular. The primary way of transmission of a pathogen into the airways is most commonly microaspiration of microbes colonizing the oropharyngeal region or the upper gastrointestinal tract ${ }^{9}$, or by droplet infection from the environment.

The most frequent pathogens causing CAP are Streptococcus pneumoniae, Haemophilus influenzae and atypical bacteria (Mycoplasma pneumoniae, Chlamydophila pneumoniae) or respiratory viruses ${ }^{12,13}$.

A 2009 pilot study carried out in our center showed that HAP was most commonly caused by endogenous pathogens originating from an individual's bacterial microflora or, to a lesser extent, by exogenous pathogens from the hospital environment with clonal spread among patients. The most frequently identified pathogens were gram-negative bacteria, in particular Klebsiella pneumoni$a e$ and Pseudomonas aeruginosa, resistant to multiple antibiotics ${ }^{5}$.

This multicenter study aimed at assessing the adequacy and effectiveness of initial antibiotic therapy of HAP with respect to mortality and providing a broader overview of the epidemiology of pathogens causing pneumonia in patients with respiratory insufficiency receiving mechanical ventilation.

\section{METHODS}

\section{Setting and Study Design}

A prospective, multicenter, observational study was designed to assess both clinical and epidemiological data of intensive care patients with CAP, HAP and VAP in the Czech Republic. The study was approved by the Ethics Committee for Multicenter Trials of the University Hospital Olomouc. Patients included in the study were not required to give informed consent. The study was entered in the ClinicalTrials.gov database under the number NCT02614144.

\section{Participants}

The following four clinical centers in the Czech Republic participated in the study: (1) Department of Anesthesiology and Intensive Care Medicine, Faculty of Medicine in Hradec Kralove, Charles University in Prague and University Hospital Hradec Kralove; (2) Department of Anesthesiology and Intensive Care Medicine, Faculty of Medicine, Masaryk University in Brno and University Hospital Brno; (3) Department of Anesthesiology and Intensive Care Medicine, First Faculty of Medicine, Charles University in Prague and Thomayer Hospital Prague; and (4) Department of Anesthesiology and Intensive Care Medicine, Faculty of Medicine and Dentistry, Palacky University Olomouc and University Hospital Olomouc.

The sample comprised patients staying in intensive care units (ICUs) of the participating centers between 1 May 2013 and 31 December 2014 who developed signs of CAP or HAP. Included were all individuals aged 18 and over who were consecutively admitted to the centers and had positive findings in their tracheal secretions during their ICU stay. In intubated patients, secretion samples for microbiology culture tests were obtained by aspiration from the lower airway. Each sample was processed by semiquantitative method based on the four-quadrant streak technique using a calibrated loop. The etiologic agent was considered relevant if cultured from tracheal secretion samples at a quantity of $>10^{5} \mathrm{CFU} / \mathrm{mL}$. The samples were processed by traditional microbiological methods. The microorganisms were identified by standard microbiological methods using MALDI-TOF mass spectrometry (Brucker Daltonics, Germany). If an identical culture, determined as the etiologic agent, was repeatedly isolated from multiple samples obtained from the same patient, only the first isolated culture was included in the study. Susceptibility to antibiotics was determined by the standard microdilution method according to the EUCAST criteria ${ }^{14}$. Production of ESBL and AmpC broad-spectrum beta-lactamases was detected by modified DDST and AmpC tests ${ }^{15}$. Positive phenotype results were verified by polymerase chain reaction detecting the blaCTX-M, blaSHV, and blaTEM genes ${ }^{16,17}$. 


\section{Outcome Assessment}

The primary endpoint was 30-day mortality. To assess relationships between mortality and the type of pneumonia, etiologic agent and adequacy of empirical antibiotic therapy, participants were divided into mono- and polymicrobial groups based on the frequency of detection of the etiologic agent. The following basic patient data were recorder: gender (male/female), age at enrolment (years), Acute Physiology and Chronic Health Evaluation II (APACHE II) score, length of hospital stay, length of mechanical ventilation and 30-day mortality. Also recorded were initial empirical antibiotic therapy on the day when the criteria for pneumonia were met and its adequacy, that is, susceptibility of the identified etiologic agent to the applied antibiotic.

\section{Definitions}

The clinical signs of pneumonia are defined as the presence of newly developed or progressive infiltrates on chest radiographs plus at least two other signs of respiratory tract infection: temperature $>38^{\circ} \mathrm{C}$, purulent sputum, leukocytosis (WBC $>10 \times 10^{3} / \mathrm{mm}^{3}$ ) or leukopenia (WBC $<4 \times 10^{3} / \mathrm{mm}^{3}$ ) signs of inflammation on auscultation, cough and/or respiratory insufficiency with a $\mathrm{PaO}_{2} / \mathrm{FiO}_{2}$ ratio of $\leq 300 \mathrm{mmHg}$.

\section{Statistical Methods}

Patients with incomplete data for the key variables at all time points were excluded from the analysis. The time point was the moment of fulfilling the criteria for pneumonia. The selection of variables for a multivariate model was based on two principles: (i) variables with $P$ $<0.1$ in univariate analysis were selected for the development of the multivariate model, and (ii) redundancy of variables was evaluated and only the most statistically significant variable out of the redundant set of predictors was selected. No replacement of missing values or outliers was performed in order to minimize bias due to changed content of retrospective clinical records. The prerequisites of statistical computations were evaluated using visual inspection of data and normality tests; the redundancy of predictors was evaluated using the analysis of their associations (correlation for continuous variables and contingency table analysis for categorical variables). Standard descriptive statistics were applied to summarize the primary data; continuous variables as means and 95\% confidence intervals or median and range; categorical variables by absolute and relative frequencies. Potential predictors of 30-day mortality were analyzed using logistic regression and risk or protective association was quantified by odds ratios (OR) with corresponding 95\% confidence intervals. Multivariate logistics regression was adopted for adjusting univariate results for age and APACHE II scores, and for defining the final multivariate model. Thirty-day mortality was statistically analyzed by Fisher's exact test. The selection of variables for the multivariate model was based on univariate $P<0.1$ and redundancy analysis of these preselected predictors. $P \leq 0.05$ was adopted as the level of statistical significance for all analyses and SPSS 21 (IBM Corporation, 2012) was the software used.

\section{RESULTS}

\section{Patients}

The group dataset comprised data on 330 enrolled patients. Of those, 116 patients were excluded as their lower airway secretion samples either yielded negative culture findings or contained normal upper airway flora. Thus, the final validated dataset involved 214 patients, 168 males (78.5\%) and 46 females (21.5\%), from whom 278 etiologic agent samples were obtained.

Community-acquired pneumonia was identified in 13 patients, from whom 18 etiologic agents were obtained (30-day mortality with adequate and inadequate initial antibiotic therapy was $0.0 \%$ and $33.3 \%$, respectively; the overall 30-day mortality reached $15.4 \%$ ).

Hospital-acquired pneumonia was identified in 201

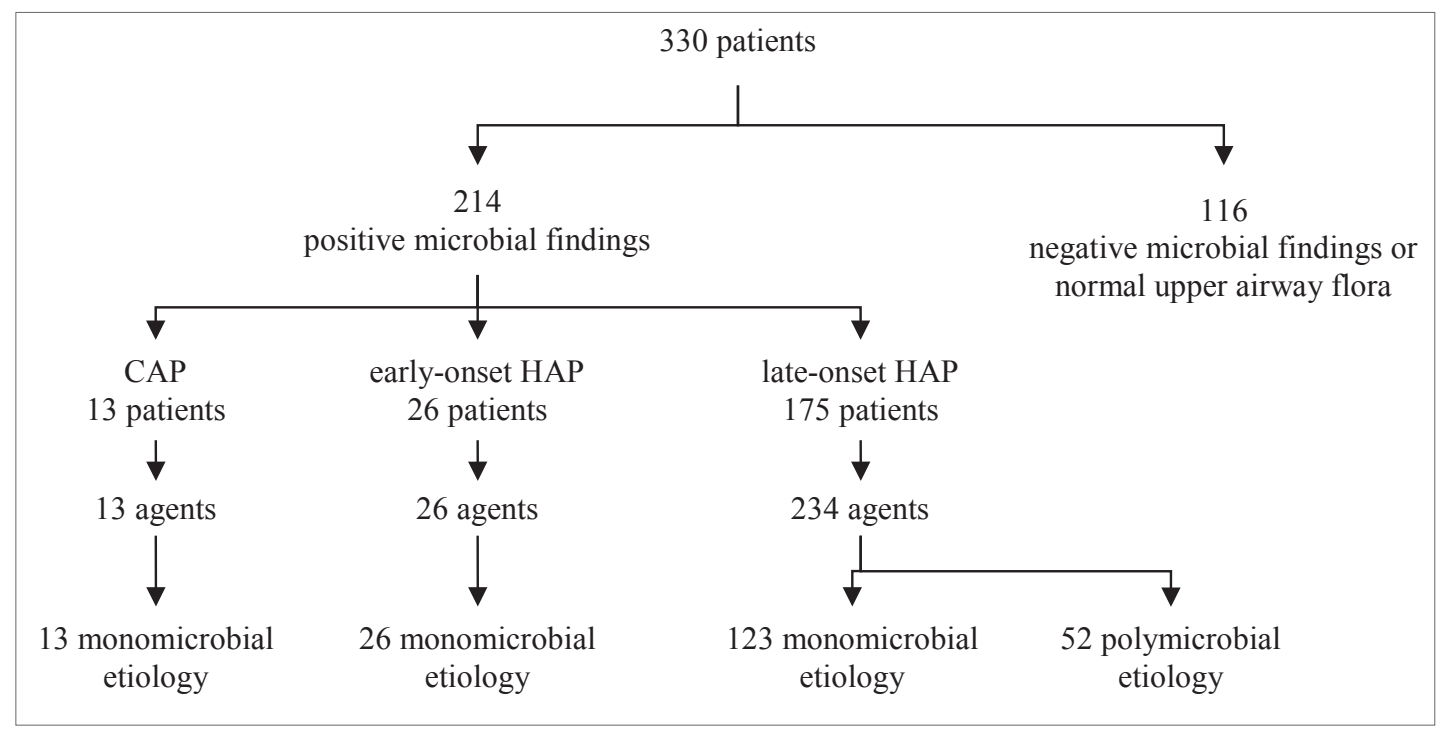

Fig. 1. Study flow chart. 
patients, from whom 260 etiologic agents were obtained. Twenty-six patients (12.9\%) were diagnosed with earlyonset HAP, with 26 samples of etiologic agents obtained. Late-onset HAP was detected in 175 patients $(87.1 \%)$ with 234 etiologic agents. Monomicrobial etiology was detected in 149 patients. Polymicrobial etiology was seen in 52 patients, all of them having late-onset HAP. The flow chart is shown in Fig. 1.

The mean patient age was $59.9 \pm 17.6$ years (median, 64.0 years); females $64.1 \pm 18.1$ years (median, 68.5 years), males $58.7 \pm 17.4$ (median, 63.0 years). Females were statistically significantly older $(P=0.021)$.

The mean APACHE II score at admission was 21.126 (range, 8-43; median, 20.0); females 22.696 (range, 8-43, median, 23.0), males 20.696 (range, 8-40; median, 20.0). The difference in scores between males and females was not statistically significant $(P=0.061)$.

\section{Main results}

A significant relationship was found between the pneumonia type and age (the mean age was 48.4, 57.5 and 60.8 years for patients with CAP, early- and late-onset HAP, respectively; $\mathrm{P}=0.049$ ). No association was found between APACHE II scores at admission and the type of pneumonia.

There was no statistically significant relationship between the type of pneumonia and patient gender $(P=$ 1.000).

Twenty-two bacterial species were identified as causing HAP (Appendix 1). Only six of them exceeded a frequency of detection of $5 \%$ (Klebsiella pneumoniae $20.4 \%$, Pseudomonas aeruginosa 20.0\%, Escherichia coli 10.8\%, Enterobacter spp. 8.1\%, Staphylococcus aureus 6.2\% and Burkholderia cepacia complex 5.8\%).

There was a statistically significant association between the etiologic agent and HAP type $(P=0.046)$. Patients infected with Staphylococcus aureus had signifi-

Table 1. Relationship between the etiologic agent and HAP type (etiologic agents from patients with monomicrobial etiology with detection rates higher than 5\%).

\begin{tabular}{|c|c|c|c|c|}
\hline & & \multicolumn{2}{|c|}{ HAP } & \multirow{2}{*}{ Total } \\
\hline & & early-onset & late-onset & \\
\hline \multirow[t]{2}{*}{ Burkholderia cepacia complex } & Count & 0 & 7 & 7 \\
\hline & $\%$ & $0.0 \%$ & $100.0 \%$ & $100 \%$ \\
\hline \multirow[t]{2}{*}{ Enterobacter spp. } & Count & 2 & 9 & 11 \\
\hline & $\%$ & $18.2 \%$ & $81.8 \%$ & $100 \%$ \\
\hline \multirow[t]{2}{*}{ Escherichia coli } & Count & 2 & 13 & 15 \\
\hline & $\%$ & $13.3 \%$ & $86.7 \%$ & $100 \%$ \\
\hline \multirow{2}{*}{ Klebsiella pneumoniae } & Count & 1 & 24 & 25 \\
\hline & $\%$ & $4.0 \%$ & $96.0 \%$ & $100 \%$ \\
\hline \multirow[t]{2}{*}{ Pseudomonas aeruginosa } & Count & 1 & 27 & 28 \\
\hline & $\%$ & $3.6 \%$ & $96.4 \%$ & $100 \%$ \\
\hline \multirow[t]{2}{*}{ Staphylococcus aureus } & Count & 5 & 8 & 13 \\
\hline & $\%$ & $38.5 \%$ & $61.5 \%$ & $100 \%$ \\
\hline \multirow[t]{2}{*}{ Total } & Count & 11 & 88 & 99 \\
\hline & $\%$ & $11.1 \%$ & $88.9 \%$ & $100 \%$ \\
\hline
\end{tabular}

Table 2. Relationship between the etiologic agent for HAP and adequate initial empirical antibiotic therapy (etiologic agents from patients with monomicrobial etiology with detection rates higher than $5 \%$ ).

\begin{tabular}{|c|c|c|c|c|}
\hline & & \multicolumn{2}{|c|}{ Adequate antibiotic therapy } & \multirow{2}{*}{ Total } \\
\hline & & Yes & No & \\
\hline \multirow[t]{2}{*}{ Burkholderia cepacia complex } & Count & 5 & 2 & 7 \\
\hline & $\%$ & $71.4 \%$ & $28.6 \%$ & $100 \%$ \\
\hline \multirow[t]{2}{*}{ Enterobacter spp. } & Count & 5 & 6 & 11 \\
\hline & $\%$ & $45.5 \%$ & $54.5 \%$ & $100 \%$ \\
\hline \multirow[t]{2}{*}{ Escherichia coli } & Count & 13 & 2 & 15 \\
\hline & $\%$ & $86.7 \%$ & $13.3 \%$ & $100 \%$ \\
\hline \multirow[t]{2}{*}{ Klebsiella pneumoniae } & Count & 16 & 9 & 25 \\
\hline & $\%$ & $64.0 \%$ & $36.0 \%$ & $100 \%$ \\
\hline \multirow[t]{2}{*}{ Pseudomonas aeruginosa } & Count & 13 & 15 & 28 \\
\hline & $\%$ & $46.4 \%$ & $53.6 \%$ & $100 \%$ \\
\hline \multirow[t]{2}{*}{ Staphylococcus aureus } & Count & 9 & 4 & 13 \\
\hline & $\%$ & $69.2 \%$ & $30.8 \%$ & $100 \%$ \\
\hline \multirow[t]{2}{*}{ Total } & Count & 61 & 38 & 99 \\
\hline & $\%$ & $61.6 \%$ & $38.4 \%$ & $100 \%$ \\
\hline
\end{tabular}


cantly higher rates of early-onset HAP than those with other etiologic agents (see Table 1).

Initial empirical antibiotic therapy included 19 different antibiotic agents as monotherapy or combination therapy (Appendix 2).

Adequate initial antibiotic therapy was noted in 120 patients (59.7\%); of those, 15 had early-onset and 105 had late-onset HAP. Adequate initial antibiotic therapy was administered to 93 patients $(62.4 \%)$ with monomicrobial etiology of HAP $(P=0.301)$ and to only 23 patients (44.2\%) with polymicrobial etiology $(P=0.219)$.

The study failed to show a relationship between the etiologic agent for HAP and adequate initial antibiotic therapy $(P=0.084)$ - see Table 2 .

\section{0-day mortality}

The overall mortality rate for HAP was $29.9 \%$, with 19.2\% mortality for early-onset HAP and $31.4 \%$ mortality for late-onset $\operatorname{HAP}(P=0.302)$.

In patients with monomicrobial etiology of HAP, the overall mortality was $28.9 \%$ and the rates for early- and late-onset HAP were $16.7 \%$ and $30.5 \%$, respectively $(P=$ 0.277 ). Those with polymicrobrobial etiology had $32.7 \%$ mortality, with the rates for early- and late-onset HAP being $28.6 \%$ and $33.3 \%$, respectively $(P=1.000)$.

The mortality rates were $21.3 \%$ and $40.2 \%$ for adequate and inadequate initial antibiotic therapy, respectively $(P$ $=0.003)$.

There was no association between mortality and the gender $(P=0.361)$ or etiologic agent causing HAP $(P=$ $0.598)$

In patients with late-onset HAP, mortality was significantly lower if adequate initial empirical antibiotic therapy was administered as compared with inadequate therapy $(P=0.012)$ - see Table 3 . In patients with early-onset HAP, however, the study failed to show the relationship between 30-day mortality and adequate initial empirical therapy $(P=0.620)$.

\section{VAP}

In 159 patients $(74.3 \%)$, HAP was identified in association with mechanical ventilation, that is, they had VAP. There was no statistically significant relationship between the development of VAP and gender $(P=0.850)$.

The subgroup of patients with VAP was shown to have statistically significantly higher mean APACHE II score (21.7) than those having HAP and not receiving mechanical ventilation $(19.3 ; P=0.031)$.

Patients with VAP $(n=55)$ had higher mortality $(34.6 \%)$ than those who developed HAP unrelated to mechanical ventilation $(\mathrm{n}=7 ; 12.7 \% ; P=0.002)$.

There was a relationship between mortality in VAP and adequate empirical antibiotic therapy. Patients having VAP treated with adequate initial antibiotic therapy had lower 30 -day mortality $(27.2 \%)$ than those receiving inadequate therapy $(44.8 \% ; P=0.028)$ - see Table 4 .

\section{DISCUSSION}

The present study was the first to collect multicenter data on the epidemiology of HAP in the Czech Republic. The result showed that the actual 30-day mortality was significantly lower than mortality predicted based on the initial APACHE II score. Patients with HAP had a real mortality rate of $30 \%$ ( $38 \%$ according to APACHE II), with the rates in the early- and late-onset HAP and VAP subgroups being 19\% (35\% APACHE II), 31\% (40\% APACHE II) and 35\% (40\% APACHE II), respectively. In the CAP patient subgroup, the actual 30-day mortality was $15 \%$, as compared with $30 \%$ according to APACHE II.

Previously published data on HAP-associated mortal-

Table 3. Mortality rates for patients with late-onset HAP receiving adequate empirical antibiotic therapy.

\begin{tabular}{|c|c|c|c|c|c|}
\hline & & & \multicolumn{2}{|c|}{ Mortality } & \multirow{2}{*}{ Total } \\
\hline & & & Yes & No & \\
\hline \multirow[t]{4}{*}{ Adequate antibiotic therapy } & Yes & Count & 25 & 80 & 105 \\
\hline & & $\%$ & $23.8 \%$ & $76.2 \%$ & $100 \%$ \\
\hline & No & Count & 30 & 40 & 70 \\
\hline & & $\%$ & $42.9 \%$ & $57.1 \%$ & $100 \%$ \\
\hline \multirow[t]{2}{*}{ Total } & & Count & 55 & 120 & 175 \\
\hline & & $\%$ & $31.4 \%$ & $68.6 \%$ & $100 \%$ \\
\hline
\end{tabular}

Table 4. Mortality rates for patients with VAP receiving adequate initial antibiotic therapy.

\begin{tabular}{|c|c|c|c|c|c|}
\hline & & & \multicolumn{2}{|c|}{ Mortality } & \multirow{2}{*}{ Total } \\
\hline & & & Yes & No & \\
\hline \multirow[t]{4}{*}{ Adequate antibiotic therapy } & Yes & Count & 25 & 67 & 92 \\
\hline & & $\%$ & $27.2 \%$ & $72.8 \%$ & $100 \%$ \\
\hline & No & Count & 30 & 37 & 67 \\
\hline & & $\%$ & $44.8 \%$ & $55.2 \%$ & $100 \%$ \\
\hline \multirow[t]{2}{*}{ Total } & & Count & 55 & 104 & 159 \\
\hline & & $\%$ & $34.6 \%$ & $65.4 \%$ & $100 \%$ \\
\hline
\end{tabular}


ity ranged between $20 \%$ and $50 \%$ (ref. ${ }^{6-9}$ ), with the most common rate being approximately $46 \%\left(\right.$ ref. $\left.^{2,18}\right)$. However, comparison of mortality data is limited by the fact that varied approaches are used to report the data. For example, Forel et al. ${ }^{19}$ assessed ICU mortality in two VAP subgroups, 28-day (27\%) and 90-day (41\%) rates, whereas Werarak reported a 30 -day mortality rate of $46 \%\left(\right.$ ref. $^{20}$ ) and Tejada et al. stated $46 \%$ mortality with no time period specified ${ }^{18}$. A study by Esperatti et al. described a group of 315 ICU patients and found in-hospital mortality rates $36 \%$ for HAP and $42 \%$ for VAP (ref. ${ }^{21}$ ). Burgmann et al. investigated nosocomial infections in 456 patients staying in 34 Austrian ICUs to report 32\% in-hospital HAP mortality $^{22}$. Rates similar to those in the present study were published in a 1-month prospective study by Hortal et al. comprising 986 patients undergoing cardiac surgery in 25 hospitals in 8 European countries; the VAP mortality was $35 \%$ (ref. ${ }^{23}$ ). By contrast, a higher VAP-associated mortality $(52 \%)$ was reported by Lambiase et al. in a smaller cohort of 29 patients ${ }^{24}$.

The present study found a 30-day HAP-associated mortality of $30 \%$, a rate corresponding with $35 \%$ in-hospital mortality reported by a 2011 pilot study by Uvizl et al. ${ }^{5}$. According to numerous studies, higher mortality rates were associated with inadequate initial antibiotic therapy, inadequate dosage, inadequate length of administration of antibiotics and/or delayed initiation of antibiotic therapy ${ }^{12,25-27}$. While $40 \%$ of patients in the present study were treated inadequately, a 2006 study by Luna et al., for example, found inadequate antibiotic therapy in as many as $68 \%$ of patients ${ }^{28}$. A Spanish study by Alvarez-Lerma et al. prospectively assessing 244 critically ill patients in 24 ICUs reported inadequate initial empirical antibiotic therapy in $14 \%$ (28\% mortality) and adequate initial antibiotic therapy in $39 \%$ of patients ( $19 \%$ mortality). However, it failed to detect the etiologic agent for HAP in $47 \%$ of patients ${ }^{29}$. A similar association between mortality and adequacy of initial antibiotic therapy was presented in a study by Rello et al. using data from 27 European ICUs, with mortality rates of $35 \%$ and $48 \%$ in patients with adequate and inadequate therapy, respectively ${ }^{30}$.

The present study showed that most (87\%) HAP cases were late-onset and caused by gram-negative bacteria, in particular Enterobacteriaceae and strains of Pseudomonas aeruginosa, Burkholderia cepacia complex and Stenotrophomonas maltophilia, accounting for $86 \%$ of cases. Similar incidence rates were reported by numerous studies ${ }^{21,23,24}$ such as an Asian study by Chung et al. involving 73 ICUs ( $82 \%$ incidence) and a study by Forel et al. $(78 \%)$ (ref. $\left.{ }^{19}\right)$.

In 98\% of cases of Pseudomonas aeruginosa being identified as a bacterial agents, the bacterium was found to cause late-onset HAP. This prevalence rate correlated with results from other studies ${ }^{2,32,33}$, namely that by Lambiase et al. reporting a prevalence of Pseudomonas aeruginosa in VAP of $52 \%\left(\right.$ ref. $\left.^{24}\right)$.

As compared with other pathogens, antibiotic therapy for Pseudomonas aeruginosa has been noted to fail due to resistance in a high proportion of cases. The present study showed inadequate therapy in $54 \%$ of cases caused by Pseudomonas aeruginosa and 39\% mortality rate when HAP was associated with isolation of this pathogen.

The detection rate of Klebsiella pneumoniae as an etiologic agent causing late-onset HAP (10\%) is comparable to previously published data ranging from $4 \%$ to $12 \%$ (ref. $^{2,19,30}$ ).

Unlike data from other studies, the role of grampositive bacteria, in particular Staphylococcus aureus, in the etiology of HAP is less pronounced in the Czech Republic. In the present study, gram-positive bacteria accounted for only $11.7 \%$ of cases, with Staphylococcus aureus being responsible for mere $7 \%$ of cases. Interestingly, Staphylococcus aureus was $100 \%$ susceptible to methicillin and the agent was associated with the second lowest mortality rate $(15 \%)$ of all pathogens investigated in the study, after Enterobacter cloacae (9\%). The proportion of Staphylococcus aureus among pathogens causing lateonset HAP was significantly lower in comparison with gram-negative bacteria. The 1997-2008 SENTRY program stated $28 \%$ prevalence of Staphylococcus aureus in the etiology of HAP; in a study by Ioanas et al., methicillinresistant strains of Staphylococcus aureus accounted for $16 \%$ of cases $^{2,34}$.

Given its low frequency, Acinetobacter baumannii could not be assessed in the present study. Yet this pathogen has been reported to have high incidence and mortality rates in association with HAP/VAP (58.8\%) (ref. ${ }^{25,33}$ ).

\section{CONCLUSIONS}

The mean age of patients with early-onset HAP (54.7 years) was lower than that of patients with late-onset HAP (60.8 years).

Pseudomonas aeruginosa was the most prevalent etiologic agent for late-onset HAP (15.4\% of cases) but it less commonly caused early-onset HAP (3.8\%). Klebsiella pneumoniae was considerably more important in the etiology of late-onset HAP (13.7\%) than in early-onset HAP (3.8\%). Staphylococcus aureus was most frequently detected in early-onset HAP (19.2\%) but less commonly in late-onset HAP (4.6\%).

The 30 -day mortality rates were $19.2 \%$ and $31.4 \%$ in patients with early- and late-onset HAP, respectively. The study failed to show relationships between mortality and type of HAP as well as between mortality and etiologic agent for HAP. Patients with late-onset HAP receiving adequate initial empirical antibiotic therapy had statistically significantly lower mortality $(23.8 \%)$ than those receiving inadequate initial antibiotic therapy (42.9\%).

Patients with VAP had significantly higher mortality (34.6\%) than patients who developed HAP with no association with mechanical ventilation $(12.7 \%)$. Patients with CAP had a mortality rate of $15.4 \%$. 


\section{ABBREVIATIONS}

APACHE II, Acute Physiology and Chronic Health Evaluation II; CAP, community-acquired pneumonia; CFU, colony-forming unit; DDST, double-disk synergy test; ESBL, extended-spectrum beta-lactamases; EUCAST, European Committee on Antimicrobial Susceptibility Testing; ICU, intensive care unit; HAP, hospital-acquired pneumonia; MALDI-TOF, matrix-assisted laser desorption/ionization time-of-flight; VAP, ventilator-associated pneumonia; WBC, white blood cell count.

Acknowledgement: The operation of the project database was supported by an unrestricted research grant from the Czech Ministry of Health (IGA MZČR NT 142633/2013).

Author contributions: TH, RU, MA, LD manuscript writing; RU, MA, TG, MHS, MK, VP: study design; TH, RU, MHS, MK, VP, KL, RZ, TR, MM, PS, JS, JM, AS, MH, ZT, PP: analysis and interpretation of data; RU, MA, MHS, MK: drafting the manuscript and revising it critically for important intellectual content; VP, KL, RZ, TR, MM, PS, JS, JM, AS, MH, ZT, PP: agreed to be accountable for all aspects of the work in ensuring that queries relating to the accuracy and integrity of the work are appropriately investigated and resolved; RU, MA, LD, MHS, MK: final approval.

Conflict of interest statement: The authors state that there are no conflicts of interest regarding the publication of this article.

\section{REFERENCES}

1. Joseph NM, Sistla S, Dutta TK, Badhe AS, Parija SC. Ventilatorassociated pneumonia: a review. Eur J Intern Med 2010;21:360-8.

2. Jones, RN. Microbial etiologies of hospital-acquired pneumonia and ventilator-associated bacterial pneumonia. Clin Index Dis 2010;51(1 Suppl):81-7.

3. Rello J, Torres A, Ricart M, Valles J, Gonzales J, Artigas A, RodriquezRoisin R. Ventilator-associated pneumonia by Staphylococcus aureus. Comparison of methicillin-resistant and methicillin-sensitive episodes. Am J Respir Crit Care Med 1994;150:1545-9.

4. Vincent JL, Bihari DJ, Suter PM, Bruining HA, White J, NicolasChanoin MH, Wolff M, Spencer RC, Hemmer M. The prevalence of nosocomial infection in intensive care units in Europe. Results of the European Prevalence of Infection in Intensive Care (EPIC) Study. EPIC International Advisory Committee. JAMA 1995 Aug 2330;274(8):639-44.

5. Uvizl R, Hanulik V, Husickova V, Htoutou Sedlakova M, Adamus M, Kolar M. Hospital-acquired pneumonia in ICU patients. Biomed Pap Med Fac Univ Palacky Olomouc Czech Repub 2011;155(4):373-8.

6. Guideline for prevention of nosocomial pneumonia. Centers for Disease Control and Prevention. Respir Care 1994;39:1191.

7. American Thoracic Society, Infectious Diseases Society of America. Guidelines for the management of adults with hospital-acquired, ventilator-associated, and healthcare-associated pneumonia. Am J Respir Crit Care Med 2005;171:388.

8. Craven DE, Palladino R, McQuillen DP. Healthcare-associated pneumonia in adults: management principles to improve outcomes. Infect Dis Clin North Am 2004;18:939.

9. Scheld WM. Developments in the pathogenesis, diagnosis and treatment of nosocomial pneumonia. Surg Gynecol Obstet 1991;172 Suppl:42.

10. Ibrahim EH, Ward S, Sherman G, Kollef MH. A comparative analysis of patients with early-onset vs late-onset nosocomial pneumonia in the ICU setting. Chest 2000;117(5):1434-42.
11. Langer M, Cigada M, Mandelli M, Mosconi P, Tognoni G. Early onset pneumonia: a multicenter study in intensive care units. Intensive Care Med 1987;13:342-6.

12. Woodhead M, Blasi F, Ewig S, Huchon G, Leven M, Ortquist A, Schaberg T, Torres A, Van der Heijden G, Verheij TJM. ERS task force in collaboration with ESCMID. Guidelines for the management of adult lower respiratory tract infections. Eur Respir J 2005;26:1138-80.

13. File TM Jr, Bartlett JG, Thorner AR. Treatment of community-acquired pneumonia in adults who require hospitalization. 2012 - pmtwww. uptodate.com

14. European Committee on Antimicrobial Susceptibility Testing. Breakpoint tables for interpretation of MICs and zone diameters. Version 1.3, 5 January 2011 [cited 27 May 2011]. Available from: http://www.eucast.org/clinical_breakpoints

15. Htoutou Sedlakova M, Hanulik V, Chroma M, Hricova K, Kolar M, Latal T, Schaumann R, Rodloff AC. Phenotypic detection of broad-spectrum beta-lactamases in microbiological practice. Med Sci Monit 2011;17:147-52.

16. Pérez-Pérez FJ, Hanson ND. Detection of plasmid-mediated AmpC $\beta$-lactamase genes in clinical isolates by using multiplex PCR. J Clin Microbiol 2002;40:2153-62.

17. Pagani L, Dell'Amico E, Migliavacca R, D'Andrea MM, Giacobone E, Amicosante G, Romero E, Rossolini GM. Multiple CTX-Mtype extended-spectrum lactamases in nosocomial isolates of Enterobacteriaceae from a hospital in northern Italy. J Clin Microbiol 2003;41:4264-9.

18. Tejada Artigas A, Bello Dronda S, Chacón Vallés E, Muñoz Marco J, Villuendas Usón MC, Figueras P, Suarez FJ, Hernández A. Risk factors for nosocomial pneumonia in critically ill trauma patients. Crit Care Med 2001;29(2):304-9.

19. Forel JM, Voillet F, Pulina D, Gacouin A, Perrin G, Barrau K, Jaber S, Arnal JM, Fathallah M, Auquier P, Roch A, Azoulay E, Papazian L. Ventilator-associated pneumonia and ICU mortality in severe ARDS patients ventilated according to a lung-protective strategy. Crit Care 2012;16(2):65.

20. Werarak P, Kiratisin P, Thamlikitkul V. Hospital-acquired pneumonia and ventilator-associated pneumonia in adults at Siriraj Hospital: etiology, clinical outcomes, and impact of antimicrobial resistance. J Med Assoc Thai 2010;93 S1:126-38.

21. Esperatti M, Ferrer $M$, Theessen A, Liapikou A, Valencia M, Saucedo LM, Zavala E, Welte T, Torres A. Nosocomial pneumonia in the intensive care unit acquired by mechanically ventilated versus nonventilated patients. Am J Respir Crit Care Med 2010;182(12):1533-9. doi: 10.1164/rccm.201001-00940C

22. Burgmann $H$, Hiesmayr JM, Savey A, Bauer P, Metnitz B, Metnitz PG. Impact of nosocomial infections on clinical outcome and resource consumption in critically ill patients. Intensive Care Med 2010;36(9):1597-601. doi: 10.1007/s00134-010-1941-2

23. Hortal J, Muñoz P, Cuerpo G, Litvan H, Rosseel PM, Bouza E. Ventilator-associated pneumonia in patients undergoing major heart surgery: an incidence study in Europe. The European Study Group of Nosocomial Infections, the European Work Group of Cardiothoracic Intensivists. Crit Care 2009;13(3):R80.

24. Lambiase A, Rossano F, Piazza O, Del Pezzo M, Catania MR, Tufano R. Typing of Pseudomonas aeruginosa isolated from patients with VAP in an intensive care unit. New Microbiol 2009;32(3):277-83.

25. Kollef MH, Sherman G, Ward S, Fraser VJ. Inadequate antimicrobial treatment of infections: a risk factor for hospital mortality among critically ill patients. Chest 1999;115(2):462-74.

26. Iregui M, Ward S, Sherman G, Fraser VJ, Kollef MH. Clinical importance of delays in the initiation of appropriate antibiotic treatment for ventilator-associated pneumonia. Chest 2002;122(1):262-8.

27. Gruson D, Hilbert G, Vargas F, Valentino R, Bebear C, Allery A, Bebear C, Gbikpi-Benissan G, Cardinaud JP. Rotation and restricted use of antibiotics in a medical intensive care unit. Impact on the incidence of ventilator-associated pneumonia caused by antibiotic-resistant gram-negative bacteria. Am J Respir Crit Care Med 2000;162:837-43

28. Luna CM, Aruj P, Niederman MS, Garzón J, Violi D, Prignoni A, Ríos F, Baquero S, Gando S. Grupo Argentino de Estudio de la Pneumonia Asociada el Respirador group. Appropriateness and delay to initiate therapy in ventilator-associated pneumonia. Eur Respir J 2006;27:158-64.

29. Alvarez-Lerma F, Alvarez B, Luque $P$, Ruiz F, Dominguez-Roldan JM, Quintana E, Sanz-Rodriguez C; ADANN Study Group. Empiric broad-spectrum antibiotic therapy of nosocomial pneumonia in 
the intensive care unit: a prospective observational study. Crit Care 2006;10(3):R78.

30. Rello J, Ulldemolins M, Lisboa T, Koulenti D, Mañez R, Martin-Loeches I, De Waele JJ, Putensen C, Guven M, Deja M, Diaz E. Determinants of prescription and choice of empirical therapy for hospital-acquired and ventilator-associated pneumonia European Respiratory Journal 2011;37:1332-9.

31. Chung RD, Song JH, Kim SH, Thamlikitkul V, Huang SG, Wang $\mathrm{H}$, Man Kit So T, Yasin RMD, Hsueh PR, Carlos CC, Yain Hsu L, Buntaran L, Lalitha MK, Kim MJ, Choi JY, Kim SI, Ko KS, Kang CI, Peck KR. High prevalence of multidrug-resistant nonfermenters in hospital-acquired pneumonia in Asia. Am J Respir Crit Care Med 2011;184(12):1409-17.
32. Crouch Brewer S, Jones CB, Leeper KV, Wunderink RG. Ventilatorassociated pneumonia due to Pseudomonas aeruginosa Chest 1996;109:1029.

33. Chastre J, Fagon JY. Ventilator-associated pneumonia. Am J Respir Crit Care Med 2002;165:867-902.

34. Ioanas M, Cavalcanti M, Ferrer M. Hospital-acquired pneumonia: coverage and treatment adequacy of current guidelines. Eur Respir J 2003;22:876-82.

\section{Supplemental Material:}

The online version of this article (doi: 10.5507/ bp.2016.014) offers supplementary material. 